\title{
Information-Distilling Quantizers
}

\section{Conference Paper}

\section{Author(s):}

Nazer, Bobak; Ordentlich, Or; Polyanskiy, Yury

Publication date:

2018-02

Permanent link:

https://doi.org/10.3929/ethz-b-000245059

Rights / license:

In Copyright - Non-Commercial Use Permitted 


\section{Information-Distilling Quantizers}

\author{
Bobak Nazer \\ $\mathrm{BU}$ \\ bobak@bu.edu
}

\author{
Or Ordentlich \\ HUJI \\ or.ordentlich@mail.huji.ac.il
}

\author{
Yury Polyanskiy \\ MIT \\ yp@mit.edu
}

Let $X$ and $Y$ be a pair of random variables with a given distribution $P_{X Y}$. This work deals with the problem of quantizing $Y$ into $M$ values, under the objective of maximizing the mutual information between the quantizer's output and $X$. We denote the value of the mutual information attained by the optimal $M$-ary quantizer by

$$
I\left(X ;[Y]_{M}\right) \triangleq \sup _{\tilde{Y} \in[Y]_{M}} I(X ; \tilde{Y}) .
$$

where $[Y]_{M}$ is the set of all (deterministic) $M$-ary quantizations of $Y$,

$$
[Y]_{M} \triangleq\{f(Y): f: \mathcal{Y} \rightarrow[M]\}
$$

When $X$ and $Y$ are thought of as the input and output of a channel, respectively, the problem boils down to designing the $M$-level quantizer that maximizes the information rate, whereas (1) is the highest information rate attainable. It is therefore not surprising that this problem has received considerable attention [1]-[5]. For example, it is well known [6, Section 2.11] that when $X$ is a BPSK input to an AWGN channel with output $Y$ it holds that $I\left(X ;[Y]_{2}\right) \geq 2 I(X ; Y) / \pi$ and this is achieved by taking $f(\cdot)$ to be the maximum a posteriori (MAP) estimator of $X$ from $Y$.

Our focus is studying the fundamental properties of the function $I\left(X ;[Y]_{M}\right)$, and in particular, identifying the joint distributions $P_{X Y}$ that are the most difficult to quantize, and characterizing the behavior of $I\left(X ;[Y]_{M}\right)$ for these cases. Special attention is given to the symmetric binary case where $X \sim \operatorname{Bernoulli}(1 / 2)$. In this setting, it may seem that the optimal binary quantizer should always retain a significant fraction of $I(X ; Y)$, and that the MAP quantizer should be sufficient to this end. For large $I(X ; Y)$, it is not difficult to see that this is indeed the case [7, Proposition 5]. However, if $Y \in\{0,1, ?\}$ is the output of a binary erasure channel with input $X$, for large erasure probabilities the MAP quantizer may be arbitrarily inferior to the asymmetric quantizer $f(0)=0$, $f(1)=f(?)=1[7$, Section III.c] .

Furthermore, in certain cases, no binary quantizer can retain a significant fraction of $I(X ; Y)$. Our main result is the following [7, Theorem 1]. Logarithms are taken w.r.t. base 2 , with the exception of the $\ln$ function that is taken w.r.t. base $e$.
Theorem 1: If $X \sim \operatorname{Bernoulli}(1 / 2)$ and $I(X ; Y)=\beta>0$, we have for binary quantization

$$
I\left(X ;[Y]_{2}\right) \geq \frac{1}{3 e} \frac{\beta}{1+\ln \left(\frac{1}{\beta}\right)} .
$$

Furthermore, for any $\eta \in(0,1)$ and any natural $M<$ $\frac{12 \max \left\{\log \left(\frac{1}{\beta}\right), 1\right\}}{(1-\eta)^{2}}$

$$
I\left(X ;[Y]_{M}\right) \geq(M-1) \frac{\beta}{\max \left\{\log \left(\frac{1}{\beta}\right), 1\right\}} \frac{\eta(1-\eta)^{2}}{12} .
$$

Finally, for any $0<\beta \leq 1$, there exist distributions $P_{X Y}$ with $X \sim \operatorname{Bernoulli}(1 / 2)$ and $I(X ; Y)=\beta$, for which

$$
I\left(X ;[Y]_{M}\right) \leq 2 M \frac{\beta}{\ln \left(\frac{e \log (e)}{2 \beta}\right)},
$$

for every natural $M$.

Note that this is in stark contrast to the intuition from the binary AWGN channel. While for the former, two quantization levels suffice for retaining a $2 / \pi$ fraction of $I(X ; Y)$, Theorem 1 shows that there exist distributions for which at least $\Omega(\log (1 / I(X ; Y)))$ quantization levels are needed in order to retain a fixed fraction of $I(X ; Y)$.

\section{REFERENCES}

[1] R. Pedarsani, S. H. Hassani, I. Tal, and E. Telatar, "On the construction of polar codes," in 2011 IEEE International Symposium on Information Theory Proceedings, July 2011, pp. 11-15.

[2] I. Tal, A. Sharov, and A. Vardy, "Constructing polar codes for nonbinary alphabets and macs," in 2012 IEEE International Symposium on Information Theory Proceedings, July 2012, pp. 2132-2136.

[3] B. M. Kurkoski and H. Yagi, "Quantization of binary-input discrete memoryless channels," IEEE Transactions on Information Theory, vol. 60, no. 8, pp. 4544-4552, Aug 2014.

[4] I. Tal, "On the construction of polar codes for channels with moderate input alphabet sizes," in IEEE International Symposium on Information Theory (ISIT), June 2015, pp. 1297-1301.

[5] A. Kartowsky and I. Tal, "Greedy-merge degrading has optimal powerlaw," arXiv preprint arXiv:1701.02119, 2017.

[6] A. J. Viterbi and J. K. Omura, Principles of digital communication and coding. Courier Corporation, 2013.

[7] B. Nazer, O. Ordentlich, and Y. Polyanskiy, "Information-distilling quantizers," in 2017 IEEE International Symposium on Information Theory (ISIT), June 2017, pp. 96-100. 